BULL. AUSTRAL. MATH. SOC.

$06 A 12,06 A 99,06 B 10$

VOL. $26(1982), 185-213$.

\title{
STANDARD ELEMENTS IN A NEARLATTICE
}

\author{
William H. CORnish and A.S.A. îoor
}

\begin{abstract}
Nearlattices, or lower semilattices in which any two elements have a supremum whenever they are bounded above, provide an interesting generalization of lattices. In this context, we study standard, neutral, and central elements, as well as standard ideals. A new perspective is obtained in the well established case of lattices.
\end{abstract}

\section{Introduction}

Standard elements and ideals in lattices were first studied in depth by Grätzer and Schmidt [8]. Since then little attention has been paid to these notions. Some additional work has been done by Janowitz [11], while Fried and Schmidt [6] have recently extended the idea of a standard ideal to a convex sublattice. As a good reference, we cite Chapter 3 of Grätzer [7].

A lower semilattice is said to have the upper bound property if the supremum of any two elements automatically exists when they share a common upper bound. Here, we follow the terminology of Cornish [3, Section 3] and Noor [13] by describing a lower semilattice with this property as a nearlattice. Alternatively, a nearlattice can be thought of as a lower semilattice $(A ; \wedge)$ such that each initial segment $(t]=\{a \in A: a \leq t\}$ is a lattice, or as a lower semilattice for which the new semilattice, that is obtained by the adjunction of a largest element, is actually a lattice. A nearlattice is distributive if each initial segment is a distributive

Received 18 March 1982. 
lattice, or equivalently if the infimum distributes over existent finite suprema.

A nearlattice-homomorphism is a semilattice-homomorphism between two nearlattices which also preserves existent finite suprema. A nearlatticecongruence $\Phi$ on a nearlattice $A$ is a congruence of the underlying lower semilattice such that, whenever $a_{1} \equiv b_{1}, a_{2} \equiv b_{2}(\Phi)$ and $a_{1} \vee a_{2}$, $b_{1} \vee b_{2}$ exist, $a_{1} \vee a_{2} \equiv b_{1} \vee b_{2}(\Phi)$. For an alternative description, see [3, Lemma 3.2]. Quite a lot of information on nearlattice-congruences is contained in the third sections of both [5] and [3]. A subset $B$ of a nearlattice $(A ; \wedge, \leq)$ is called a subnearlattice if $(B ; \wedge)$ is a subsemilattice of $(A ; \wedge)$ such that, whenever $c=a \vee b$ of $a, b, c \in B$, $c$ is also the supremum of $a$ and $b$ in $A$.

In the second section of [10], a fundamental contribution was made by Hickman. He showed that the category of nearlattices $(A ; \wedge, \leq)$ and their nearlattice-homomorphisms is isomorphic to a variety of ternary algebras $(A ; j)$ and their homomorphisms, in such a way that isomorphism commutes with the forgetful functors which ground these categories in the category of sets. Moreover, the isomorphism takes subnearlattices to subalgebras and nearlattice-congruences to congruences of the corresponding ternary algebra. These ternary algebras were called join algebras by Hickman and his ternary operation $j$ is given by $j(x, y, z)=(x \wedge y) \vee(x \wedge z)$ on any nearlattice. Its existence is ensured by the upper bound property; actually, Hickman preferred $(x \wedge y) \vee(y \wedge z)$ for his ternary operation.

Hickman's work shows that nearlattices or join algebras can be regarded as the subreducts of lattices $(L ; \wedge, \vee)$ with respect to the derived operation $j(x, y, z)=(x \wedge y) \vee(x \wedge z)$. For a simplification in the case of distributive lattices and also the subreducts of lattices with respect to the operation $x \wedge(y \vee z)$, see Baker [1] and Cornish [4].

Most importantly, Hickman's work enables us to consider free nearlattices. In particular, the free nearlattice $F N(x, y, z)$ on three generators $x, y, z$ can be found inside the free lattice $F L(x, y, z)$ as the j-subalgebra generated by $x, y, z$. The lattice $F L(x, y, z)$ is extremely complex; it is pictured in [7, p. 283]. However, $F N(x, y, z)$ has only ten elements, namely $x, y, z, j(x, y, z), j(y, x, z), j(z, x, y)$, $x \wedge y, y \wedge z, z \wedge x$, and $x \wedge y \wedge z$. Its diagram is given in Hickman 
[10, Figure 2, p. 1668]; it also occurs in a slightly different context in Baker [1, p. 143]. It should be observed that the nearlattice $F(x, y, z)$ is distributive. Hence, any three-generated nearlattice is distributive!

The upshot of this last observation is that at least three arbitrary elements must be involved in any first order descriptions which extend the notions of standard and neutral elements from lattices to nearlattices. For, the very conceptions of standard and neutral elements in a lattice are concerned with the distributive behaviour of these special elements and two other elements.

In Section 2, we extend the notions of standard element and standard ideal to a nearlattice. The emphasis is on the behaviour of the congruences associated with them. The congruence $\theta_{s}$, associated with a standard element $s$ of a nearlattice $A$, can be neatly described in terms of the j-operation by:

$$
\begin{aligned}
& x \equiv y\left(\Theta_{s}\right) \text { if and only if, for all } t \in A, \\
& (t \wedge x) \vee(t \wedge s)=(t \wedge y) \vee(t \wedge s) .
\end{aligned}
$$

Section 3 is concerned with neutral and central elements. Section 4 deals with characterizations of these special elements, $x$, in terms of the properties of their traces, $t \wedge x$, in initial segments, $(t]$. In this way, we are led to the notion of a strongly distributive element. Also, it turns out that an element $n$ of a nearlattice, or lattice, is neutral if and only if, for all $t, x$, and $y$,

$(t \wedge n \wedge x) \vee(t \wedge n \wedge y) \vee(t \wedge x \wedge y)$

$$
=((t \wedge n) \vee(t \wedge x)) \wedge((t \wedge n) \vee(t \wedge y)) \wedge((t \wedge x) \vee(t \wedge y)) \text {. }
$$

Thus, in the process of generalizing the notions of standard element et cetera, and their properties to nearlattices, we obtain new insights and results about these notions in the case of lattices. In writing this paper, the authors have in mind the application of the idea of a neutral element to the study of isotopes of nearlattices; a summary of part of that study can be found in Noor [13]. Also, nearlattices occur naturally in the investigation of reducts of certain algebraic structures, see for example [4] and [3, Section 3], and the notion of a standard ideal might well prove useful there. 


\section{Standard elements and ideals}

Recall that an element $s$ of lattice is standard if $x \wedge(y \vee s)=(x \wedge y) \vee(x \wedge s)$, for all $x$ and $y$. There are important equivalents of this definition. For ease of comparison, we summarize them in the following proposition; for proofs, see Grätzer and Schmidt [8] and Grätzer [7, Theorem 3, p. 139].

PROPOSITION 2.1. The following conditions zpon an element $s$ of a lattice $L$ are equivalent.

\section{(i) $s$ is standard.}

(ii) The relation $\theta_{s}$, defined by $x \equiv y\left(\theta_{s}\right)$ if and only if $x \vee y=(x \wedge y) \vee s_{1}$ for some $s_{1} \leq s$, is a congmence relation.

(iii) $s$ is a distributive element, that is $s \vee(x \wedge y)=(s \vee x) \wedge(s \vee y)$ for cony $x, y \in L$, and $b=c$ whenever $s \wedge b=s \wedge c$ and $s \vee b=s \vee c$.

(iv) For each ideal $K$, (s] $\vee K=\left\{s_{1} \vee k: s_{1} \leq s, k \in K\right\}$.

(v) (s] is a stondard element of the ideal lattice of $L$.

An element $s$ of a nearlattice $A$ is called standard if for all $x, y, t \in A$,

$$
t \wedge((x \wedge y) \vee(x \wedge s))=(t \wedge x \wedge y) \vee(t \wedge x \wedge s) .
$$

(Notice that both sides exist because of the upper bound property.)

Obviously, any element of a distributive nearlattice is standard. Now suppose $\boldsymbol{s}$ is asstandard element of a lattice $L$. Then, for all $x, y, t \in L$,

$$
t \wedge((x \wedge y) \vee(x \wedge s))=t \wedge x \wedge(y \vee s)=(t \wedge x \wedge y) \vee(t \wedge x \wedge s) \text {. }
$$

This and a part of the next proposition show that the two concepts coincide in a lattice.

PROPOSITION 2.2. The following two conditions on an arbitrary element $s$ of a nearlattice $A$ are equivalent.

(i) For any $x, y \in A, x \wedge(y \vee s)=(x \wedge y) \vee(s \wedge x)$ whenever $y \vee s$ exists. 
(ii) (a) If $x \vee s$ and $y \vee s$ exists for any $x, y \in S$, then $(x \wedge y) \vee s$ exists and $(x \wedge y) \vee s=(x \vee s) \wedge(y \vee s)$;

(b) for any $x, y \in A$, for which $x \vee s$ and $y \vee s$ exist, $x \wedge s \geq y \wedge s$ and $x \vee s \geq y \vee s$ imply $x \geq y$.

Moreover, both (i) and (ii) are necessary for $s$ to be standard but are not sufficient.

Proof. (i) $\Rightarrow(i i)$. Suppose $x, y \in A$ are such that $x \vee s$ and $y \vee s$ exist. Then $(x \wedge y) \vee s$ exists because of the upper bound property. Due to $(i)$, $(x \vee s) \wedge(y \vee s)=[(x \vee s) \wedge y] \vee[(x \vee s) \wedge s]$ $=(x \wedge y) \vee(s \wedge y) \vee s=(x \wedge y) \vee s$.

Also, if $x \wedge s \geq y \wedge s$ and $x \vee s \geq y \vee s$, then.

$$
\begin{aligned}
x=x \wedge(x \vee s) & \geq x \wedge(y \vee s)=(x \wedge y) \vee(x \wedge s) \quad(\text { by }(i)) \\
& \geq(x \wedge y) \vee(y \wedge s)=y \wedge(x \vee s) \quad(\text { by }(i)) \\
& \geq y \wedge(y \vee s)=y .
\end{aligned}
$$

(ii) $\Rightarrow$ (i). Suppose $x, y \in A$ and $y \vee s$ exists. Let

$p=x \wedge(y \vee s)$ and $q=(x \wedge y) \vee(x \wedge s)$. Now

$$
p \wedge s=x \wedge s \leq q=(x \wedge y) \vee(x \wedge s) \leq x \wedge(y \vee s)=p .
$$

Hence $p \wedge s \leq q \wedge s \leq p \wedge s$, that is $p \wedge s=q \wedge s$. Observe that as $p, s \leq y \vee s, p \vee s$ exists and since $p=p \wedge(y \vee s)$,

$$
\begin{aligned}
p \vee s & =[p \wedge(y \vee s)] \vee s \\
& =(p \vee s) \wedge(y \vee s) \quad(b y(i i)(a)) \\
& =(p \wedge y) \vee s \quad(b y(i i)(a)) \\
& =(x \wedge y) \vee s \\
& =(x \wedge y) \vee(x \wedge s) \vee s \\
& =q \vee s .
\end{aligned}
$$

Then by (ii) (b), $p=q$, that is, (i) holds.

Now suppose $s$ is standard in $A, x, y \in A$ and $y \vee s$ exists. Then letting $y \vee s=r$ we obtain $x \wedge(y \vee s)=x \wedge[(r \wedge y) \vee(r \wedge s)]$

$$
=(x \wedge r \wedge y) \vee(x \wedge r \wedge s)=(x \wedge y) \vee(x \wedge s) \text {, }
$$

as $s$ is standard; thus $(i)$ and $(i i)$ hold. 
Finally, consider the nearlattice $S$ in Figure 1 . Here, for all $x, y \in A$, the condition (i) holds; but $d \wedge[(c \wedge a) \vee(c \wedge s)]>(d \wedge c \wedge a) \vee(d \wedge c \wedge s)$.

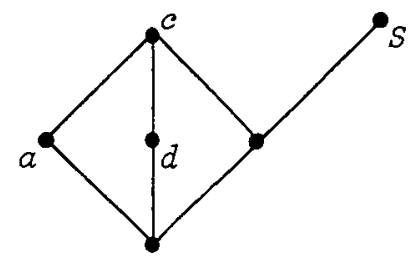

FIGURE 1

The following lemma is useful when verifying that a binary relation is a nearlattice-congruence; it is an extension of a characterization of lattice-congruences; cf. Grätzer [7, Lemma 8, p. 20] and Grätzer and Schmidt [8].

LEMMA 2.3. A reflexive, symetric binary relation $\theta$ on a nearlattice $A$ is a nearlattice-congmence if and only if, for any $x, y, z, t \in A$,

$$
\begin{aligned}
& \text { (i) } x \equiv y(\theta) \text { if and only if } x \wedge y \equiv x(\theta) \text { and } x \wedge y \equiv y(\theta), \\
& \text { (ii) } x \leq y \leq z, x \equiv y(\theta) \text { and } y \equiv z(\theta) \text { imply } x \equiv z(\theta), \\
& \text { (iii) } x \leq y \text { and } x \equiv y(\theta) \text { imply that } x \wedge t \equiv y \wedge t(\theta) \text { and } \\
& x \vee t \equiv y \vee t(\theta), \text { whenever } x \vee t \text { cond } y \vee t \text { exist. }
\end{aligned}
$$

Proof. The only if part is trivial. So assume that a reflexive and symmetric relation $\theta$ satisfies conditions $(i)-(i i i)$.

To prove the transitivity of $\theta$, suppose $x \equiv y(\theta)$ and $y \equiv z(\theta)$ so that $x \wedge y \equiv x(\theta), x \wedge y \equiv y(\theta), y \wedge z \equiv y(\theta)$ and $y \wedge z \equiv z(\theta)$. Then by (iii), $x \wedge y \wedge z \equiv y \wedge z(\theta)$. But $y \wedge z \equiv z(\theta)$ and so by (ii), $x \wedge y \wedge z \equiv z(\theta)$. Also, $x \wedge y \wedge z \leq x \wedge z \leq z$ and so by (iii), $x \wedge z \equiv z(\theta)$. Similarly, $x \wedge z \equiv x(\theta)$. Then by $(i), x \equiv z(\theta) ; \theta$ is transitive and so an equivalence relation.

Suppose $x \equiv y(\theta)$ so that $x \wedge y \equiv x(\theta)$ and $x \wedge y \equiv y(\theta)$. By i iiiz), $x \wedge y \wedge t \equiv x \wedge t(\theta)$ and $x \wedge y \wedge t \equiv y \wedge t(\theta)$ for any $t \in S$. Thus $x \wedge t \equiv y \wedge t(\theta)$ by transitivity. It follows that $\Theta$ has the substitution property for $\wedge$.

Suppose $x \equiv y(\theta)$ and $x \vee t, y \vee t$ both exist for some $t \in S$. 
Clearly, $(x \wedge y) \vee t$ exists. Now $x \wedge y \equiv x(\theta)$ and $x \wedge y \equiv y(\theta)$. Hence, by (iii), $(x \wedge y) \vee t \equiv x \vee t(\theta)$ and $(x \wedge y) \vee t \equiv y \vee t(\theta)$. Consequently, $x \vee t \equiv y \vee t(\theta)$. Finally, suppose $x \equiv y(\theta)$ and $x_{1} \equiv y_{1}(\theta)$ and $x \vee x_{1}, y \vee y_{1}$ exist. Then $x \equiv x \wedge y \equiv y(\theta)$ and so $x \vee x_{1} \equiv(x \wedge y) \vee x_{1} \equiv(x \wedge y) \vee y_{1} \equiv y \vee y_{1}(\theta)$.

Before proceeding to our first main result, we recall the notion of a nearlattice-ideal, or simply an ideal. An ideal $K$ of a nearlattice $A$ is a non-empty subset such that

$$
\begin{aligned}
& \text { (i) } x \in K \text { if } x \leq k \in K \text {, and } \\
& \text { (ii) } k_{1} \vee k_{2} \in K \text { whenever } k_{1} \vee k_{2} \text { exists for given } \\
& k_{1}, k_{2} \in K .
\end{aligned}
$$

The ideals of a nearlattice form a lattice. Set-intersection is the infimum. The supremum is, in general, awkward to work with. If $J$ and $K$ are ideals of $A, S_{0}=J \cup K$, and

$S_{n}=\left\{x \in A: x \leq y \vee z ; y \vee z\right.$ exists and $\left.y, z \in S_{n-1}\right\}$ for $n=1,2, \ldots$, then

$$
J \vee K=\bigcup_{n=0}^{\infty} S_{n} .
$$

In effect, this is a case of Exercise 22 in Grätzer [7, p. 45]; it has an easy inductive proof. Of course, the initial segment $(x)$ is the smallest ideal containing $x$.

THEOREM 2.4. For an element $s$ of a nearlattice $A$ the following conditions are equivalent.

(i) $s$ is a standard element.

(ii) The binary relation $\theta$, which is defined by $x \equiv y(\theta)$ if and only if $x=(x \wedge y) \vee(x \wedge s)$ and $y=(x \wedge y) \vee(y \wedge s)$, is a nearZattice-congruence.

(iii) The binary relation $\Phi$, which is defined by $x \equiv y(\Phi)$ if and only if $(x \wedge t) \vee(t \wedge s)=(y \wedge t) \vee(t \wedge s)$ for all $t \in A$, is a nearlattice-congruence. 
(iv) For each ideal $K$,

(s] $\vee K=\left\{s_{1} \vee k: s_{1} \leq s, k \in K\right.$ and $s_{1} \vee k$ exists $\}$.

(v) (s] is a standard element of the ideal lattice of $A$. Moreover, $\Theta$ and $\Phi$ of $(i i)$ and (iii), respectively, represent the same join-partial congruence, namely $\theta_{s}$, the smallest nearlattice-congruence of $A$ having ( $s]$ as a congruence class.

Proof. $(i) \Rightarrow$ (ii). Let $\theta$ be the binary relation such that $x \equiv y(\theta)$ if and only if $x=(x \wedge y) \vee(x \wedge s)$ and $y=(x \wedge y) \vee(y \wedge \varepsilon)$. Clearly, $\theta$ is reflexive and symmetric. Now $x \equiv y(\theta)$ implies

$$
x=(x \wedge y) \vee(x \wedge s)=(x \wedge(x \wedge y)) \vee(x \wedge s) .
$$

Also,

$$
x \wedge y=(x \wedge(x \wedge y)) \vee((x \wedge y) \wedge s),
$$

and so $x \equiv x \wedge y(\theta)$. Similarly, $y \equiv x \wedge y(\theta)$. Conversely, $x \wedge y \equiv x(\theta)$ and $x \wedge y \equiv y(\theta)$ certainly imply $x \equiv y(\theta)$. Suppose $x \leq y \leq z$ and $x \equiv y(\theta), y \equiv z(\theta)$. Then $z=y \vee(z \wedge s)$ and $y=x \vee(y \wedge s)$. So

$$
z=x \vee(y \wedge s) \vee(z \wedge s)=x \vee(z \wedge s)
$$

and it follows that $x \equiv z(\theta)$.

Now let $x \leq y, x \equiv y(\theta)$ and $x \vee t, y \vee t$ exist for some $t \in A$. Then $y \vee t=[x \vee(y \wedge s)] \vee t=(x \vee t) \vee(y \wedge s)$; that is $y \vee t=(x \vee t) \vee[(y \vee t) \wedge s]$, which implies $x \vee t \equiv y \vee t(\theta)$. Also, for any $r \in A$,

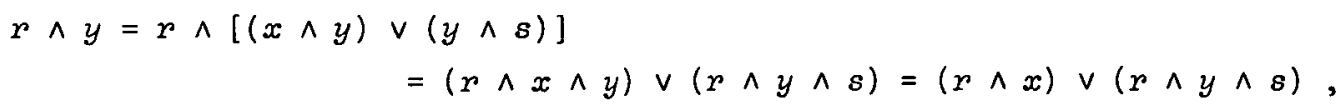

and so $r \wedge y \equiv r \wedge x(\theta)$. Due to Lemma 2.3, $\theta$ is a nearlatticecongruence.

$$
\text { (ii) } \Rightarrow \text { (iii). Suppose } x \equiv y(\theta) \text {. Since } \theta \text { is a join-partial }
$$

congruence, $x \wedge t \equiv y \wedge t(\theta)$ for any $t \in A$. Then

$$
x \wedge t=(x \wedge y \wedge t) \vee(x \wedge t \wedge s)
$$

and 


$$
y \wedge t=(x \wedge y \wedge t) \vee(y \wedge t \wedge s)
$$

and hence

$$
(x \wedge t) \vee(t \wedge s)=(x \wedge y \wedge t) \vee(t \wedge s)=(y \wedge t) \vee(t \wedge s)
$$

this implies that $x \equiv y(\Phi)$. Conversely, let $x \equiv y(\Phi)$. Then $(x \wedge t) \vee(t \wedge s)=(y \wedge t) \vee(t \wedge s)$ for all $t \in A$. By letting $t=x$ and $t=y$ we obtain $x=(x \wedge y) \vee(x \wedge s)$ and $y=(x \wedge y) \vee(y \wedge s)$ respectively. Hence $x \equiv y(\Phi)$. This implies that $\Theta$ and $\Phi$ are the one and the same nearlattice-congruence.

$$
\begin{aligned}
& (i i i) \Rightarrow(i v) \text {. The set } \\
& \quad T=\left\{s_{1} \vee k: s_{1} \leq s, k \in K \text { and } s_{1} \vee k \text { exists }\right\}
\end{aligned}
$$

is clearly closed under existent finite suprema. Suppose $x \leq s_{1} \vee k$ with $s_{1} \leq s$ and $k \in K$. Clearly, $s_{1} \vee k=k(\Phi)$ and so $x=x \wedge\left(s_{1} \vee k\right) \equiv x \wedge k(\Phi)$. Hence for all $t \in A$,

$$
(x \wedge t) \vee(t \wedge s)=(x \wedge k \wedge t) \vee(t \wedge s) .
$$

Choosing $t=x$ we obtain $x=(x \wedge k) \vee(x \wedge s)$ and so $x \in T$. Thus $T$ is the ideal of $A$ and it is clearly the supremum of ( $s$ ] and $K$.

$(i v) \Rightarrow(v)$. Let $J$ and $K$ be two ideals of $A$ and suppose $x \in J \cap((s] \vee K)$. Then $x \in J$ and $x=s_{1} \vee k$ for some $s_{1} \leq s$ and $k \in K$. So $x=\left(x \wedge s_{1}\right) \vee(x \wedge k)$ and thus $x \in(J \cap(s]) \vee(J \cap K)$. Consequently, $J \cap((s] \vee K)=(J \cap(s]) \vee(J \cap K)$, which implies that (s] is standard in the ideal lattice of $S$.

(v) $\Rightarrow$ (i) is trivial.

The last part is quite clear in view of the proof of $(i i) \Rightarrow$ (iii) and the definition of $\theta$.

We now turn to ideals. An ideal $K$ in a nearlattice $A$ is standard if it is a standard element of the ideal-lattice $J(A)$. Of course, Theorem 2.4 says $s$ is a standard element if and only if (s) is a standard ideal.

THEOREM 2.5. Let $K$ be an ideal in a nearlattice. Then the following conditions are equivalent. 
(i) $K$ is a standard ideal.

(ii) The binary relation $\Theta(K)$, defined by $x \equiv y(\Theta(K))$ if and only if $x=(x \wedge y) \vee(x \wedge a), y=(x \wedge y) \vee(y \wedge b)$ for some $a, b \in K$, is a nearlattice-congruence.

(iii) The binary relation $\Phi$, defined by $x \equiv y(\Phi)$ if and only if for all $t \in S,(x \wedge t) \vee(t \wedge c)=(y \wedge t) \vee(t \wedge c)$ for some $c \in K$, is a nearlattice-congruence.

(iv) For each ideal $H$, $K \vee H=\{k \vee h: k \vee h$ exists and $k \in K$ and $h \in H\}$.

Moreover, (ii) and (iii) represent the same nearlattice-congruence, namely $\Theta(K)$, the smallest join-partial congruence of $A$ having $K$ as a congruence class.

Proof. $(i) \Rightarrow(i i)$. Due to condition $(i)$ the relation $J \equiv H\left(\Theta_{K}\right)$ $(J, H \in J(A))$ if and only if $J=(J \cap H) \vee(J \cap K)$ and $H=(J \cap H) \vee(H \cap K)$ is a congruence on $J(A)$. Then $\Theta_{K \mid A}$ (restriction to $A$ ) is a nearlattice-congruence on $A$ and $x \equiv y\left(\Theta_{K \mid A}\right)$ if and only if $(x]=(x \wedge y] \vee((x] \cap K)$ and $(y]=(x \wedge y] \vee((y] \cap K)$. Thus to prove (ii), it is sufficient to prove that $(x]=(x \wedge y] \vee((x] \cap k)$ implies $x=(x \wedge y) \vee(x \wedge a)$ for some $a \in K$. Now

$$
(x \wedge y] \vee((x] \cap K)=\bigcup_{n=0}^{\infty} A_{n},
$$

where $A_{0}=(x \wedge y] \cup((x] \cap K)$ and $A_{n}=\left\{t \in S \mid t \leq p \vee q ; p \vee q\right.$ exists and $\left.p, q \in A_{n-1}\right\}$ for $n=1,2, \ldots$ : and we show, by induction, that

$$
(x \wedge y] \vee((x] \cap K)=\{t: t \leq(x \wedge y) \vee(x \wedge a) \text { for some } a \in K\} .
$$

If $t \in A_{0}$ then $t \in(x \wedge y]$ or $t \in(x] \cap K$. In the first instance, $t \leq x \wedge y \leq(x \wedge y) \vee(x \wedge k)$ for any $k \in K$ and in the second instance, $t=t \wedge x \leq(x \wedge y) \vee(x \wedge t)$ and $t \in K$. Thus the result holds for $n=0$. Suppose the result holds for $n-1$ for some $n \geq 1$. Let $t \in A_{n}$. Then $t \leq p \vee q$ with $p, q \in A_{n-1}$. So $p \leq(x \wedge y) \vee\left(x \wedge k_{1}\right)$ 
and $q \leq(x \wedge y) \vee\left(x \wedge k_{2}\right)$ for some $k_{1}$ and $k_{2} \in K$. Then $t \leq(x \wedge y) \vee\left(x \wedge k_{1}\right) \vee\left(x \wedge k_{2}\right)=(x \wedge y) \vee(x \wedge k)$ for some $k \in K$ (since $\left(x \wedge k_{1}\right) \vee\left(x \wedge k_{2}\right) \leq x$ and is in $K$, it is of the form $x \wedge k$ for some $k \in K$ ). Thus we have

$(x \wedge y] \vee((x] \cap K)=\{t: t \leq(x \wedge y) \vee(x \wedge k)$ for some $k \in K\}$; in effect, $x \leq(x \wedge y) \vee(x \wedge a)$ for some $a \in K$ and so $x=(x \wedge y) \vee(x \wedge a)$, as required.

(ii) $\Rightarrow$ (iii). Let $x \equiv y(\Theta(K))$. Since $\Theta(K)$ is a congruence, $x \wedge t \equiv y \wedge t(\Theta(K))$ for any $t \in A$, and so

$$
x \wedge t=(x \wedge y \wedge t) \vee(x \wedge t \wedge a)
$$

and

$$
y \wedge t=(x \wedge y \wedge t) \vee(y \wedge t \wedge b)
$$

for some $a, b \in K$. Then

$$
\begin{aligned}
(x \wedge t) \vee(t \wedge[(t \wedge a) \vee(t \wedge b)]) & =(x \wedge t) \vee(t \wedge a) \vee(t \wedge b) \\
=(x \wedge y \wedge t) \vee(t \wedge a) & \vee(t \wedge b)=(y \wedge t) \vee(t \wedge a) \vee(t \wedge b) \\
& =(y \wedge t) \vee(t \wedge[(t \wedge a) \vee(t \wedge b)]) .
\end{aligned}
$$

Observe that $(t \wedge a) \vee(t \wedge b) \in K$. Thus $x \equiv y(\Phi)$.

Conversely, if $x \equiv y(\Phi)$ then for any $t \in A$,

$$
(x \wedge t) \vee(t \wedge c)=(y \wedge t) \vee(t \wedge c) \text { for some } c \in K \text {. }
$$

Choosing $t=x$ and $t=y$, we have $x=(x \wedge y) \vee(x \wedge a)$ and $y=(x \wedge y) \vee(y \wedge b)$ respectively, for some $a, b \in K$. Thus $x \equiv y(\Theta(K))$ and $\Phi$ is the nearlattice-congruence $\Theta(K)$.

$$
\begin{aligned}
& (\text { iii) } \Rightarrow(\text { iv) } \text {. Let } \\
& \qquad T=\{k \vee h: k \vee h \text { exists and } k \in K, h \in H\} .
\end{aligned}
$$

Suppose $x \leq k \vee h, k \in K, h \in H$. Clearly $k \vee h \equiv h(\Theta(K))$ and so $x=x \wedge(k \vee h) \equiv x \wedge h(\Theta(K))$. Hence, for all $t \in S$,

$$
(x \wedge t) \vee(t \wedge c)=(x \wedge h \wedge t) \vee(t \wedge c) \text { for some } c \in K \text {. }
$$

Choosing $t=x$, we obtain $x=(x \wedge h) \vee(x \wedge b)$ and so $x \in T$. But $T$ is closed under existent finite suprema. It follows that $T$ is closed under existent finite suprema. It follows that $T$ is an ideal of $S$ and 
$T=K \vee H$.

(iv) $\Rightarrow$ (i). Let $x \in J \cap(K \vee H)$. Then $x \in J$ and $x \in K \vee H$. So $x=k \vee h$ for suitable $k \in K$ and $h \in H$. Then $x=(x \wedge k) \vee(x \wedge h)$ and so $x \in(J \cap K) \vee(J \cap H)$. The reverse inclusion is obvious. Thus $J \cap(K \vee K)=(J \cap K) \vee(J \cap H) ; K$ is a standard ideal.

The final assertion is clear in view of the proof of (ii) $\Rightarrow$ (iii).

The next result looks at the collection of all standard elements.

THEOREM 2.6. The standard elements of a nearlattice $A$ form $a$ distributive subnearlattice of $A$. Moreover, the map $s \mapsto \Theta_{s}$ is a nearlattice embedding of this subnearlattice into the distributive lattice of all nearlattice-congruences on $A$.

Proof. Let $s$ and $t$ be standard elements.

Suppose $x \equiv y\left(\theta_{s} \cap \theta_{t}\right)$. Then $x \wedge s \equiv x \wedge y \wedge s\left(\theta_{t}\right)$ and by Theorem 2.4, $x \wedge s=(x \wedge y \wedge s) \vee(x \wedge s \wedge t)$. But $x=(x \wedge y) \vee(x \wedge s)$ and so $x=(x \wedge y) \vee(x \wedge s \wedge t)$. Similarly, $y=(x \wedge y) \vee(y \wedge s \wedge t)$. Conversely, these last two equalities clearly imply $x \equiv y\left(\Theta_{s} \cap \theta_{t}\right)$. It follows from Theorem 2.4 that $s \wedge t$ is standard and what is more $\Theta_{s \wedge t}=\theta_{s} \cap \theta_{t}$. Here our method of proving $s \wedge t$ is standard is the same as that of Grätzer and Schmidt [8] or Grätzer [7, Theorem 9, p. 143], but we believe our details are essentially simpler.

Suppose $s \vee t$ exists in $A$. For $x, y, z \in A$, $z \wedge[(x \wedge y) \vee(x \wedge(s \vee t))]$

$$
\begin{aligned}
& =z \wedge[(x \wedge y) \vee(x \wedge s) \vee(x \wedge t)] \text { (by Proposition 2.2) } \\
& =z \wedge[(x \wedge((x \wedge y) \vee(x \wedge s))] \vee(x \wedge t)] \\
& =(z \wedge x \wedge((x \wedge y) \vee(x \wedge s))) \vee(x \wedge z \wedge t) \\
& =(z \wedge x \wedge y) \vee(z \wedge x \wedge s) \vee(z \wedge x \wedge t) \\
& =(z \wedge x \wedge y) \vee(t \wedge x \wedge(s \vee t)),
\end{aligned}
$$

by a second application of Proposition 2.2. Hence $s \vee t$ is standard. It is now clear that the set of all standard elements form a distributive subnearlattice of $A$.

It remains to show that $\theta_{s} \vee \theta_{t}=\theta_{s \vee t}$, when $s \vee t$ exists. Now 
$\theta_{s} \vee \theta_{t} \subseteq \theta_{s \vee t}$. Hence, suppose $x \equiv y\left(\Theta_{s \vee t}\right)$. Then $x=(x \wedge y) \vee(x \wedge(s \vee t))=(x \wedge y) \vee(x \wedge s) \vee(x \wedge t)$.

Also $(x \wedge y) \vee(x \wedge s) \vee(x \wedge t) \equiv(x \wedge y) \vee(x \wedge t)\left(\Theta_{s}\right)$ and $(x \wedge y) \vee(x \wedge t) \equiv x \wedge y\left(\Theta_{t}\right)$. Hence $x \equiv x \wedge y\left(\Theta_{s} \vee \Theta_{t}\right)$. Similarly, $y \equiv x \wedge y\left(\Theta_{s} \vee \Theta_{t}\right)$. Then $x \equiv y\left(\Theta_{s} \vee \theta_{t}\right)$, as required.

From Theorem 2.6 we can obtain the expected result about standard ideals. Indeed, the proof of Theorem 2.5 shows that for any standard ideal $K$ of a nearlattice $A, \Theta(K)=\Theta_{K} \mid A$, the restriction of the congruence $\Theta_{K}$ on the ideal-lattice $J(A)$ to its subnearlattice of principal ideals. For any standard ideals, $H$ and $K$ it is clear that $\left(\Theta_{H} \mid A\right) \cap\left(\Theta_{K} \mid A\right)=\theta_{H \cap K} \mid A$. Of course, $H \vee K$ is standard, and if $x \equiv y(\Theta(J \vee K))$ then $x=(x \wedge y) \vee(x \wedge(h \vee k))$ for some $h \in H$ and $k \in K$, due to $(i v)$ of Theorem 2.5. As $h \vee k \equiv k(\Theta(H))$, we see that $\Theta_{H \vee K} \mid A \subseteq\left(\Theta_{H} \mid A\right) \vee\left(\Theta_{K} \mid A\right)$. Hence $\left(\Theta_{H} \mid A\right) \vee\left(\Theta_{K} \mid A\right)=\Theta_{H \vee K} \mid A$, also. Combining this with Theorem 2.6, we obtain

COROLLARY 2.7. The standard ideals of a nearlattice $A$ form a distributive sublattice of the ideal-Zattice $J(A)$ and the map $K \mapsto \theta(K)$ is a lattice-embedding of this sublattice into the distributive lattice of all nearlattice-congruences on $A$.

Of course, results like the above corollary generalize corresponding ones about lattices. However, they cannot be directly inferred from the case of lattices. Indeed, the ideal-lattice of a nearlattice need not be isomorphic to the ideal-lattice of any lattice. This is because Hickman [10, pp. 1676-1677] has produced a nearlattice in which the intersection of two finitely generated ideals is not finitely generated.

The next result might well be important in future applications; it generalizes both a part of [5, Theorem 3.6] and [7, Theorem 10, p. 149]. It is a modification of Grätzer's proof, [7], via (ii) of Theorem 2.5.

THEOREM 2.8. Let $A$ be a nearlattice with a smallest element 0 in which each initial segment is a complemented lattice. Then the map $K \mapsto \Theta(K)$ is a lattice-isomorphism of the lattice of standard ideals of $A$ onto the lattice of nearlattice-congmences of $A$. 
Proof. Let $\Phi$ be a nearlattice-congruence of $A$ and $J=\{x \in A: x \equiv O(\Phi)\}$. Of course, $J$ is an ideal. Suppose $a \equiv b(\Phi)$ and let $c$ and $d$ be respective complements of $a \wedge b$ in $(a]$ and (b]. Then $c=c \wedge a \equiv c \wedge a \wedge b=0(\Phi)$ and $d=d \wedge b \equiv 0(\Phi)$. Also $a=(a \wedge b) \vee(a \wedge c)$ and $b=(a \wedge b) \vee(b \wedge d)$ with $c, d \in J$. Conversely, these last relations imply $a \equiv b(\Phi)$. Hence, by Theorem 2.5, $J$ is a standard ideal and $\Phi=\theta(J)$. The remainder follows from Corollary 2.7.

The situation is more complex when it comes to permutability. We close this section with some results in this direction.

A lower semilattice $(A ; \wedge)$ is called medial if the supremum $(x \wedge y) \vee(y \wedge z) \vee(z \wedge x)$ exists for all $x, y, z \in A$. This is equivalent to saying that the supremum of any three elements exists when the suprema of each pair exist. Thus, a medial lower semilattice is a nearlattice and so will be referred to as a medial nearlattice.

THEOREM 2.9. Let $s$ and $t$ be standard elements of a medial nearlattice $A$. Then $\theta_{s}$ and $\theta_{t}$ are permutable if and only if $s \vee t$ exists.

Proof. Let $\Theta_{s}$ and $\theta_{t}$ be permutable. Since $s \equiv s \wedge t\left(\Theta_{s}\right)$ and $s \wedge t \equiv t\left(\Theta_{t}\right)$, there exists $x \in A$ such that $s=(s \wedge x) \vee(s \wedge t)$, $x=(s \wedge x) \vee(x \wedge t)$ and $t=(x \wedge t) \vee(t \wedge s)$. Let $r=(s \wedge t) \vee(t \wedge x) \vee(x \wedge s)$. Then $s, t \leq r$ and so $s \vee t$ exists.

Conversely, suppose $s \vee t$ exists and suppose $x \equiv y\left(\Theta_{s}\right)$ and $y \equiv z\left(\Theta_{t}\right)$, so that $x=(x \wedge y) \vee(x \wedge s), y=(x \wedge y) \vee(y \wedge s)$, $y=(y \wedge z) \vee(y \wedge t)$ and $z=(y \wedge z) \vee(z \wedge t)$. Let $p=(x \wedge y \wedge z) \vee(x \wedge s) \vee(z \wedge t) ; p$ exists as each pair formed from $x \wedge y \wedge z, x \wedge s$ and $z \wedge t$ has a supremum, due to the existence of $s \vee t$. But $z \wedge t \equiv x \wedge y \wedge t\left(\Theta_{t}\right)$ and $y \equiv z\left(\Theta_{t}\right)$. Hence

$$
p \equiv(x \wedge y) \vee(x \wedge s) \vee(x \wedge y \wedge t)=(x \wedge y) \vee(x \wedge s)=x\left(\Theta_{t}\right) .
$$

Also $x \equiv y\left(\Theta_{s}\right)$ and $x \wedge s \equiv y \wedge z \wedge s\left(\theta_{s}\right)$ imply that

$$
p \equiv(y \wedge z) \vee(y \wedge z \wedge s) \vee(z \wedge t)=(y \wedge z) \vee(z \wedge t)=z\left(\Theta_{8}\right)
$$


It now follows that $\theta_{s}$ and $\theta_{t}$ permute.

COROLLARY 2.10. The following conditions on a distmibutive medial nearlattice $A$ are equivalent.

(i) $A$ is a distributive lattice.

(ii) For any two ideals $J$ and $K, \Theta(J)$ and $\Theta(K)$ are permutable.

(iii) For any $s, t \in S, \theta_{s}$ and $\theta_{t}$ are permutable.

Proof. When $A$ is distributive, all of its ideals and elements are standard. Hence the corollary follows from Theorem 2.9 and the well known fact that suprema of mutually permuting congruences also permute. See also [7, Theorem 9, p. 149].

Finally, we would like to point out that the medial property is essential for Theorem 2.9. For example, consider the distributive nearlattice $N$ which is obtained by omitting the largest element from the 8-element Boolean lattice with maximal elements $a, b$, and $c$. Let its smallest element be 0 and its minimal elements be $e=b \wedge c$, $f=a \wedge b$, and $g=c \wedge a$. Actually, $N$ is the smallest nearlattice which is not medial. However, $\Theta_{a}$ has as its partition $\{\{0, f, g, a\},\{b, e, c\}\}$ and $\theta_{b}$ has as its partition $\{\{0, e, f, b\},\{g, a, c\}\}$, so that it is not hard to check that the product $\theta_{a} \circ \theta_{b}$ is the largest congruence of $N$. Thus $\theta_{a}$ and $\theta_{b}$ are permutable and yet $a \vee b$ does not exist.

\section{Neutral and central elements}

LEMMA 3.1. The following conditions on an element $d$ of a nearlattice $A$ are equivalent.

(i) For all $t, x, y \in A$,

$$
d \wedge((t \wedge x) \vee(t \wedge y))=(d \wedge t \wedge x) \vee(d \wedge t \wedge y) \text {. }
$$

(ii) For all $x, y \in A$ for which $x \vee y$ exists,

$$
d \wedge(x \vee y)=(d \wedge x) \vee(d \wedge y) \text {. }
$$

(iii) For all ideals $J$ and $K$ of $A$, 


$$
(d] \cap(J \vee K)=((d] \cap J) \vee((d] \cap K) \text {. }
$$

(iv) The binary relation $\Psi_{d}$, defined by $x \equiv y\left(\Psi_{d}\right)$ if and only if $x \wedge d=y \wedge d$, is a nearlattice-congmence.

Proof. $(i) \Rightarrow$ (ii). When $x \vee y$ exists, put $t=x \vee y$ in (i) to obtain (ii).

$(i i) \Rightarrow(i i i)$. Let $x \in(d] \cap(J \vee K)$. Then $x \leq d$ and $x \in S_{m}$ for some $m=0,1,2, \ldots$, where $S_{0}=J \cup K$ and

$$
S_{m}=\left\{t \in A: t \leq a \vee b ; a \vee b \text { exists; } a, b \in S_{m-1}\right\} \text {, }
$$

when $m \geq 1$. Suppose $x \in S_{0}$. Then $x \in(d] \cap J$ or $x \in(d] \cap K$, and so $x \in((d] \cap J) \vee((d] \cap K)$. Now we will proceed by induction. Suppose $y \in S_{m-1}$ and $y \leq d$ implies that $y \in((d] \cap J) \vee((d] \cap K)$. Since $x \in S_{m}, x \leq a \vee b$ for suitable $a, b \in S_{m-1}$. Then

$$
x \leq d \wedge(a \vee b)=(d \wedge a) \vee(d \wedge b) .
$$

But $d \wedge a, d \wedge b \leq d$ and both belong to $S_{m-1}$. Hence $x \in((d] \cap J) \vee((d] \cap K)$. The reverse inclusion is always true.

$$
(\text { iii }) \Rightarrow(i) \text { is trivial. }
$$

Also, the equivalence of (ii) and (iv) is easy to see.

With respect to this lemma and Proposition 2.2 and its associated Figure 1, we note that the element $n$ of the nearlattice $S$ of Figure 2, below, satisfies the equivalent conditions of Lemma 3.1 , as well as the condition that $x \wedge(y \vee n)=(x \wedge y) \vee(x \wedge n)$ whenever $y \vee n$ exists, and yet it is not standard. Indeed, from Figure 2, we see that $b \wedge((c \wedge a) \vee(c \wedge n))=b>0=(b \wedge c \wedge a) \vee(b \wedge c \wedge n)$.

If $A$, in Lemma 3.1, is actually a lattice, then an element $d$, satisfying the equivalent conditions of this lemma, would normally be referred to as being dually distributive, of. Grätzer [7, p. 138]. But, in the context of nearlattices, there does not appear to be a satisfactory notion of a distributive element; see Section 4, herein, and especially our idea of a strongly distributive element. Thus, we propose to call an element $d$ of a nearlattice distribuant if it satisfies the equivalents of 
Lemma 3.1.

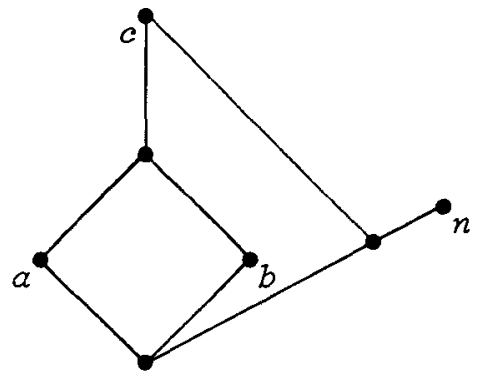

FIGURE 2

LEMMA 3.2. An element $n$ of nearlatice $A$ is both standard and distribuant if and only if there is a nearlattice-embedding (which can be taken to be subdirect) into a direct product $B \times C$ nearlattices, where $B$ has a largest element $I$ (and so is a lattice) and $C$ has a smallest element 0 , such that $n$ is mapped to $(1,0)$.

Proof. When $n$ is standard and distribuant, we can take the embedding as the subdirect decomposition $A \rightarrow A / \Psi_{n} \times A / \Theta_{n}$, by virtue of Theorem 2.4 and Lemma 3.1. The converse has an easy, but enjoyable, computational proof. However, we omit the details.

Let us call an element $n$ of a nearlattice neutral if it satisfies the equivalent conditions of Lemma 3.2. This must be a controversial choice! However, as a first defence, we have

THEOREM 3.3. An element $n$ of a nearlattice $A$ is neutral if and only if $(n)$ is neutral in the ideal-lattice $J(A)$, even when $J(A)$ is considered as a lattice and neutral is interpreted in its usual latticetheoretic sense.

Proof. This is because of Propositions 2.1, 2.2, Theorem 2.4, Lemma 3.1, and Grätzer's Theorem 3, Theorem 4 [7, pp. 139-140].

Still, how should we define a neutral element? The classical notion in Lattice Theory is of an element such that it and any other two elements generate a distributive sublattice, see Birkhoff [2, p. 69]. This is of no use in Nearlattice Theory due to our remarks in Section 1! More recently, Grätzer [7, p. 138] has proposed that an element $n$ of lattice $L$ be defined to be neutral when 


$$
(n \wedge x) \vee(n \wedge y) \vee(x \wedge y)=(n \vee x) \wedge(n \vee y) \wedge(x \vee y)
$$

for any $x, y \in L$; this is because of an elegant result proved independently by Grätzer, lqbalunissa, and also Hashimoto and Kinugawa; see the remark at the bottom of p. 140 in [7] for references. Of course, this is not directly helpful to us, but we do give a supplement to it in Section 4. The only other helpful possibilities that are supplied to us by Lattice Theory are the lattice-versions of Lemma 3.2 and Theorem 3.3. Of course, our nearlattice-definition takes these into account. This is our third defence, if we allude to our remarks about Grätzer's definition as our second defence. The lattice-version of Lemma 2.2 occurs in Birkhoff [2, p. 69] and it is skilfully exploited by Hájek in [9], which incidentally is not referred to in the exhaustive bibliography to [7].

Fourthly, we should also require a central element to be neutral. We shall presently see that this is the case. Finally, our concept of neutrality should have applications; this is so, due to [13].

Having finished this discussion, we can proceed to state the following easy consequence of Theorem 2.6 and a painless computation.

\section{THEOREM 3.4. The neutral elements of a nearlattice form a} distributive subnearlattice.

We now turn to central elements. It is customary to define the centre of a poset only when it has bounds; $c f$. Birkhoff [2, pp. 66-67]. However, Kolibiar [12] seems to be the first to have ridded us of the fear of bounds, at least in the case of lattices. Therefore, we define an element $z$ of a poset $A$ to be central if there is an order-isomorphism of $A$ onto the direct product $B \times C$ of posets $B$, with a largest element 1 , and $C$, with a smallest element 0 , such that $z$ is carried to the element $(1,0)$. With this notation, it is routinely verifiable that an element $z$ of a nearlattice $A$ is central if and only if there is a nearlattice-isomorphism of $A$ onto the direct product $B \times C$ of a lattice $B$, with a largest element $I$, and a nearlattice $C$ with a smallest 0 , such that $z$ is mapped to $(1,0)$. This provides our definition of a central element, and the subset $Z(A)$ of all central-elements in $A$ is called the centre of $A$.

Before stating the next result, we define an element $u$ of a nearlattice $A$ to be upper if $u \vee x$ always exists for any $x \in A$. 
THEOREM 3.5. The following conditions on an element $z$ of a nearlattice $A$ coincide.

(i) $z$ is central.

(ii) $z$ is neutral and upper, and complemented in each interval, which contains it.

(iii) $z$ is standard and upper, and complemented in each interval, which contains it.

Proof. $\quad(i) \Rightarrow(i i)$. Suppose (i) holds. Due to Lemma $3.2, z$ is neutral: and the remainder is not hard to obtain.

(ii) $\Rightarrow$ (i). As $z$ is upper, standard and distribuant, Proposition 2.2 and Lemma 3.1 imply that the map $\phi: A \rightarrow(z] \times[z)$, where $[z)$ is the subnearlattice $\{t \in A: t \geq z\}$, is a nearlattice homomorphism. Also $\phi$ is one-to-one. But $\phi$ is onto as $(a, b) \epsilon(z] \times[z)$ says that $a \leq z \leq b$, and so $(a, b)=\phi(c)$, where $c$ is the relative complement of $z$ in the interval $[a, b]$. Thus $\phi$ is an isomorphism and it does the required thing for $z$. Hence $z$ is central.

(i) $\Rightarrow$ (iii) is obvious.

(iii) $\Rightarrow$ (ii). Suppose (iii)holds and $x, y, t \in A$. Consider the interval $[(x \wedge y \wedge z) \vee(t \wedge x \wedge z), z \vee(x \wedge y) \vee(x \wedge t)]$, which certainly contains $z$. Let $r$ be the complement of $z$ in this interval. From Proposition 2.2, we infer that

$$
\begin{aligned}
x \wedge y=(x \wedge y) \vee[z \vee(x & \wedge y) \vee(x \wedge t)] \\
& =(x \wedge y) \vee(r \vee z)=(x \wedge y \wedge r) \vee(x \wedge y \wedge z) .
\end{aligned}
$$

Similarly,

$$
x \wedge t=(x \wedge t \wedge r) \vee(x \wedge t \wedge z)
$$

Thus

$$
\begin{aligned}
(x \wedge y) \vee(x \wedge t)=(x \wedge y \wedge r) & \vee(x \wedge t \wedge r) \vee(x \wedge y \wedge r) \vee(x \wedge t \wedge z) \\
& =(x \wedge y \wedge r) \vee(x \wedge t \wedge r) \vee(r \wedge z) \leq r
\end{aligned}
$$

and so

$$
z \wedge[(x \wedge y) \vee(x \wedge t)] \leq r \wedge z=(x \wedge y \wedge z) \vee(t \wedge x \wedge z) .
$$

Therefore 


$$
z \wedge[(x \wedge y) \vee(x \wedge t)]=(z \wedge x \wedge y) \vee(z \wedge x \wedge t)
$$

which says that $z$ is also distribuant and, therefore, neutral.

From the above theorem, it is clear that a nearlattice is a relatively complemented distributive lattice if and only if each of its elements is central. Another immediate consequence of Theorem 3.5 is

COROLLARY 3.6. In a lattice, any element which is standard and complemented in each interval containing it is actually neutral, and so central.

This corollary is an improvement upon Grätzer and Schmidt [8, Corollary 3, p. 45], namely, in a relatively complemented lattice, each standard element is neutral; $c f$. the combined effect of [7, Corollary 9, p. 134] and [7, Corollary 8, p. 143]. We know of no reference to Corollary 3.6 .

THEOREM 3.7. The centre $Z(A)$ of a nearlatice $A$ is a relatively complemented distributive lattice and a subnearlattice of $A$.

Proof. Let $z_{1}, z_{2} \in Z(A)$. Due to Theorem 3.5, $z_{1} \vee z_{2}$ exists: and it is clear that $z_{1} \wedge z_{2}$ and $z_{1} \vee z_{2}$ are neutral. Also, as each of $z_{1}$ and $z_{2}$ is upper, both $z_{1} \wedge z_{2}$ and $z_{1} \vee z_{2}$ are upper. If $[a, b]$ is an interval which contains either $z_{1} \wedge z_{2}$ or $z_{1} \vee z_{2}$, then the interval $J=\left[a \wedge z_{1} \wedge z_{2}, b \vee z_{1} \vee z_{2}\right]$ contains all of $a, b, z_{1}, z_{2}$, $z_{1} \wedge z_{2}$ and $z_{1} \wedge z_{2}$. Moreover, $J$ is a bounded lattice and $z_{1}, z_{2}$ are central elements of this lattice. Hence, their complements in $J$ are central and the infimum and supremum of these complements can be suitably compressed to produce the desired complements of $z_{1} \wedge z_{2}$ or $z_{1} \vee z_{2}$ in $[a, b]$, as the case may be. It only remains to show that $Z(A)$ is relatively complemented. We now give the details.

Suppose $z, z_{1}, z_{2} \in Z(A)$ are such that $z_{1} \leq z \leq z_{2}$. Let $z^{\prime}$ be the element of $A$ such that $z \wedge z^{\prime}=z_{1}$ and $z \vee z^{\prime}=z_{2}$. Since $z^{\prime}$ is beneath the upper element $z_{2}$, the upper bound property ensures that $z^{\prime}$ is an upper element. 
As $z, z_{1}, z_{2}$ are neutral,

$$
\begin{aligned}
t \wedge\left((x \wedge y) \vee\left(x \wedge z^{\prime}\right)\right) \wedge z & =t \wedge\left((x \wedge y \wedge z) \vee\left(x \wedge z \wedge z^{\prime}\right)\right) \\
& =t \wedge((x \wedge y \wedge z) \vee(x \wedge z)) \\
& =(t \wedge x \wedge y \wedge z) \vee\left(t \wedge x \wedge z_{1}\right) \\
& =(t \wedge x \wedge y \wedge z) \vee\left(t \wedge x \wedge z^{\prime} \wedge z\right) \\
& =\left((t \wedge x \wedge y) \vee\left(t \wedge x \wedge z^{\prime}\right)\right) \wedge z .
\end{aligned}
$$

Similarly,

$$
\left(t \wedge\left((x \wedge y) \vee\left(x \wedge z^{\prime}\right)\right) \vee z\right)=\left((t \wedge x \wedge y) \vee\left(t \wedge x \wedge z^{\prime}\right)\right) \vee z .
$$

Because of Proposition 2.2,

$$
t \wedge\left((x \wedge y) \vee\left(x \wedge z^{\prime}\right)\right)=(t \wedge x \wedge y) \vee\left(t \wedge x \wedge z^{\prime}\right) \text {. }
$$

In other words, $z^{\prime}$ is standard. In a similar way, we can show that $z^{\prime}$ is distribuant. Thus $z^{\prime}$ is neutral.

Finally, suppose $a \leq z^{\prime} \leq b$. Then $a \wedge z_{1} \leq z_{1}, z_{2} \leq b \vee z_{2}$ and there exist $p, q \in A$ such that $a \wedge z_{1}=p \wedge z_{1}=q \wedge z_{2}$ and

$$
\begin{aligned}
b \vee z_{2}=p \vee z_{1} & =q \vee z_{2} . \text { Let } p=(((p \wedge z) \vee q) \wedge b) \vee a \text {. Then } \\
r \wedge z^{\prime} & =\left(\left(\left(p \wedge z \wedge z^{\prime}\right) \vee\left(q \wedge z^{\prime}\right)\right) \wedge\left(b \wedge z^{\prime}\right)\right) \vee\left(a \wedge z^{\prime}\right) \\
& =\left(\left(\left(p \wedge z_{1}\right) \vee\left(q \wedge z^{\prime}\right)\right) \wedge z^{\prime}\right) \vee a \\
& =\left(\left(\left(a \wedge z_{1}\right) \vee\left(q \wedge z^{\prime}\right)\right) \wedge z^{\prime}\right) \vee a \\
& =\left(q \wedge z^{\prime}\right) \vee a .
\end{aligned}
$$

But $q \wedge z^{\prime} \leq q \wedge z_{2}=a \wedge z_{1} \leq a$. Thus $r \wedge z^{\prime}=a$. Similarly, we show that $r \vee z^{\prime}=b$. That is, $z^{\prime}$ is complemented in $[a, b]$, and so is central. The proof is complete.

\section{Traces}

We first introduce a new type of element and then, in our attempts to understand it, we are led to new characterizations of our previous elements via the notion of trace.

PROPOSITION 4.1. Let $A$ be a nearlattice and $s \in A$. Then the following two conditions are equivalent.

(i) For any $x, y, t \in A$, 
$(t \wedge x \wedge y) \vee(t \wedge s)=((t \wedge x) \vee(t \wedge s)) \wedge((t \wedge y) \vee(t \wedge s))$.

(ii) For any $x, y, t \in A$,

$$
(t \wedge((x \wedge y) \vee(x \wedge s))) \vee(x \wedge s)=(t \wedge x \wedge y) \vee(x \wedge s) \text {. }
$$

Proof. $(i) \Rightarrow(i i)$. Suppose (i) holds. Let $p=(x \wedge y) \vee(x \wedge s)$. Then $p \wedge x=p$ and so

$(t \wedge((x \wedge y) \vee(x \wedge s))) \vee(x \wedge s)$

$=(t \wedge p) \vee(x \wedge s)$

$=(x \wedge t \wedge p) \vee(x \wedge s)$

$=((x \wedge t) \vee(x \wedge s)) \wedge((x \wedge p) \vee(x \wedge s))$

(by (i); here $x, t$ and $p$ play the roles of $t, x$ and $y$,

$=((x \wedge t) \vee(x \wedge s)) \wedge((x \wedge y) \vee(x \wedge s))$

respectively)

$=(x \wedge t \wedge y) \vee(x \wedge s)$, by a second application of $(i)$,

where $x, t$ and $y$ play the respective roles of $t, x$, and $y$.

(ii) $\Rightarrow$ (i). Suppose (ii) holds. Then

$$
\begin{aligned}
((t \wedge x) \vee(t \wedge s)) \wedge & ((t \wedge y) \vee(t \wedge s)) \\
& =(((t \wedge x) \vee(t \wedge s)) \wedge(t \wedge y) \wedge(t \wedge s))) \vee(t \wedge s) \\
& =(((t \wedge x) \vee(t \wedge s)) \wedge(t \wedge y)) \vee(t \wedge s)
\end{aligned}
$$

(by $(i i)$, where $(t \wedge x) \vee(t \wedge s), t$ and $y$ play the roles of $t, x$ and $y$, respectively). Hence

$$
\begin{aligned}
& ((t \wedge x) \vee(t \wedge s)) \wedge((t \wedge y) \vee(t \wedge s)) \\
& =(((t \wedge x) \vee(t \wedge s)) \wedge(t \wedge y)) \vee(t \wedge s)=(y \wedge t \wedge x) \vee(t \wedge s),
\end{aligned}
$$

by a second application of (ii), where $y, t$ and $x$ play the roles of $t$, $x$ and $y$, respectively.

Now suppose $A$ of the above proposition is actually a lattice and $s \in A$ satisfies the equivalent conditions. Let $a, b \in A$ and put $a \vee b \vee s=t$ to obtain

$$
\begin{aligned}
(a \wedge b) \vee s & =(t \wedge a \wedge b) \vee(t \wedge s) \\
& =((t \wedge a) \vee(t \wedge s)) \wedge((t \wedge b) \vee(t \wedge s)) \\
& =(a \vee s) \wedge(b \vee s),
\end{aligned}
$$

so that $s$ is what is customarily called a distributive element of the lattice $A$. 
Thus we shall call an element of a nearlattice strongly distributive if it satisfies the equivalent conditions of Proposition 4.1. It is clear that a standard element is strongly distributive. Now let us look at the lattice of Figure 3, below; this lattice has been used by Grätzer [7, p. 144] to show that the infimum $a \wedge b$ is not distributive even though both $a$ and $b$ are distributive. In Figure $3, b$ is distributive and $a$ is strongly distributive. However

$$
(a \wedge t \wedge h) \vee(a \wedge b)<((a \wedge t) \vee(a \wedge b)) \wedge((a \wedge h) \vee(a \wedge b)),
$$

so $b$ is not strongly distributive. In addition,

$$
b \wedge(a \vee c)>(b \wedge a) \vee(b \wedge c)
$$

and so $a$ is not standard.

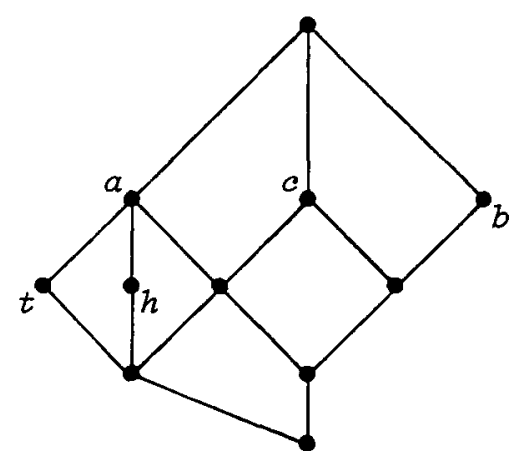

FIGURE 3

It is not hard to show that any distributive atom of a lattice with a smallest element is actually strongly distributive. Thus the atom of the pentagon, which is covered by the largest element, is strongly distributive but is not standard.

Thus, even for lattices, the notion of a strongly distributive element is strictly between the concepts of distributive and standard element. How is it possible to form a better understanding of this notion?

Let $s$ be an element of a nearlattice $A$. If $t$ is any given element of $A$, then by the trace of $s$ in (t] or more simply, the trace of $\varepsilon$, when the element $t$ is understood, we mean the element $t \wedge s$ of $(t]$. Perhaps it should be emphasized that the trace of an element is $a$ member of a lattice and so lattice-theoretic ideas and results can be applied to it. 
In this direction, part ( $i$ ) of Proposition 4.1 says an element is strongly distributive if and only if its traces are always distributive. This observation is useful when checking whether an element is strongly distributive. In view of the example associated with Figure 3, it is also fair enough to say that, for lattices, the notion of a distributive element is not trace-invariant! What can be said about the trace-invariance of the other notions of this paper? They are all trace-invariant provided that we only deal with an upper element when considering a central element.

THEOREM 4.2. Let $s$ be an element of a nearlattice $A$. Then

(i) $s$ is distribuant if and only if all of its traces are distribuant,

(ii) $s$ is standard if and only if all of its traces are standard,

(iii) $s$ is neutral if and only if all of its traces are neutral,

(iv) $s$ is strongly distributive if and only if alz of its traces are strongly distributive,

(v) provided that $s$ is an upper element, $s$ is central if and only if all of its traces are central.

Proof. (i) This is an immediate consequence of part (i) of Lemma 3.1 .

(ii) Suppose $s$ is standard and $a, b \in(t]$. Then $a \wedge(b \vee(t \wedge s))=a \wedge((t \wedge b) \vee(t \wedge s))$

$$
=(a \wedge b \wedge t) \vee(a \wedge(t \wedge s))=(a \wedge b) \vee(a \wedge(t \wedge s)),
$$

and so $t \wedge s$ is a standard element of the lattice $(t]$.

Conversely, suppose each trace is standard. As $x \wedge s$ is standard in $(x], t \wedge((x \wedge y) \vee(x \wedge s))=(t \wedge x) \wedge((x \wedge y) \vee(x \wedge s))$ must equal $((t \wedge x) \wedge(x \wedge y)) \vee((t \wedge x) \wedge(x \wedge s))=(t \wedge x \wedge y) \vee(t \wedge x \wedge s)$.

Thus $s$ is standard.

(iii) This follows from (i) and (ii).

(iv) If $s$ is strongly distributive, then using part (i) of Proposition 4.1, it is easily checked that $t \wedge s$ satisfies the same condition in $(t)$. Hence, each trace is strongly distributive. 
Conversely, if each trace $t \wedge s$ is strongly distributive, then it is also distributive in the lattice $(t]$. Hence $s$ is strongly distributive.

(v) Suppose $s$ is central. By (iii), $t \wedge s$ is neutral in ( $t]$. Now suppose $p \leq t \wedge s \leq q \leq t$. As $s$ is an upper element $q \vee s$ exists, and $p \leq s \leq q \vee s$. Let $r$ be the complement of $s$ in $[p, q \vee s]$. Then it is not hard to show that $t \wedge r$ is the complement of $t \wedge s$ in $[p, q]$. Thus each trace of $s$ is central.

The converse is trivial, provided that we assume that $s$ is upper. This is because of ( $i$ ii). The assumption that $s$ is upper is necessary otherwise every element in a nearlattice, with a smallest element and the property that each initial segment is a Boolean lattice, would be central.

This theorem appears to be trivial, yet it does have an interesting consequence.

COROLLARY 4.3. An element $n$ of a nearlatice $A$ is neutral if and only if, for alz $t, x, y \in A$,

$$
\begin{aligned}
(t \wedge n \wedge x) \vee(t \wedge n \wedge y) \vee(t \wedge x \wedge y) \\
\quad=((t \wedge n) \vee(t \wedge x)) \wedge((t \wedge n) \vee(t \wedge y)) \wedge((t \wedge x) \vee(t \wedge y)) .
\end{aligned}
$$

Proof. When $n$ is neutral, its trace $t \wedge n$ is neutral in the lattice $(t]$ and so the equality holds as $t \wedge n, t \wedge x$ and $t \wedge n$ then generated a distributive sublattice of $(t]$. Conversely, the equality says that $t \wedge n$ is neutral in the lattice $(t]$, due to [7, Theorem 4, p. 140]. Then Theorem 4.2 does the rest.

We conclude this paper with two observations about strongly distributive elements.

THEOREM 4.4. Let $L$ be a lattice and $s \in L$. Then the following conditions are equivalent.

(i) $s$ is strongly distributive in $L$.

(ii) (s] is strongly distributive in the ideal-lattice $J(L)$ of $L$.

But (i) does not necessarily imply (ii) when $L$ is merely a nearZattice.

Proof. Suppose $s$ is strongly distributive. Let $I, J$ and $K$ be 
ideals of $L$. Suppose

$$
x \in[(I \cap J) \vee(I \cap(s])] \cap[(I \cap K) \vee(I \cap(s])] \text {. }
$$

Then $x \leq p_{1} \vee q_{1}, p_{2} \vee q_{2}$ for some $p_{1} \in I \cap J, p_{2} \in I \cap K$ and $q_{1}, q_{2} \in I \cap(s]$. Let $r=p_{1} \vee p_{2} \vee q_{1} \vee q_{2}$. Clearly $r \in I$ and $q_{1}, q_{2} \leq r \wedge s$. Hence

$x \leq\left[p_{1} \vee(r \vee s)\right] \wedge\left[p_{2} \vee(r \wedge s)\right]$

$$
\begin{aligned}
& =\left[\left(p_{1} \wedge r\right) \vee(r \wedge s)\right] \wedge\left[\left(p_{2} \wedge r\right) \vee(r \wedge s)\right] \\
& =\left(p_{1} \wedge p_{2} \wedge r\right) \wedge(r \wedge s)=\left(p_{1} \wedge p_{2}\right) \vee(r \wedge s) .
\end{aligned}
$$

Thus $x \in(I \cap J \cap K) \vee(I \cap(s])$. As the reverse inclusion always holds, (ii) is established. The converse, $(i i) \Rightarrow(i)$, is trivial.

As to our final assertion, consider the nearlattice $L$ of Figure 4. There $s$ is strongly distributive as its traces are always distributive. Observe that $J(L)$ is as given in Figure 5. Nevertheless,

$$
(I \cap J \cap K) \vee(I \cap(s])<[(I \cap J) \vee(I \cap(s])] \cap[(I \cap K) \vee(I \cap(s])]
$$

in the notation of Figure 5. Consequently (s] is not strongly distributive in $J(L)$.

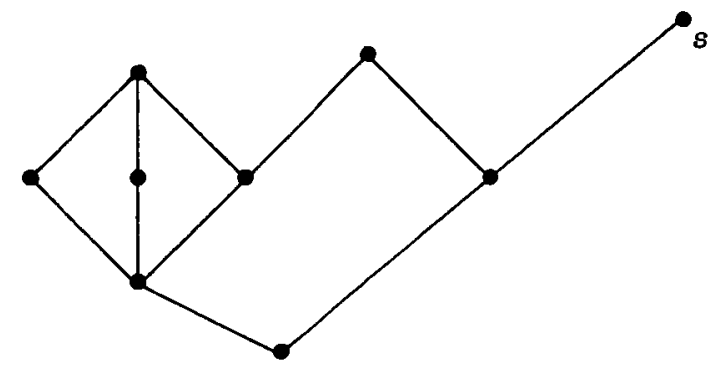

FIGURE 4

Lastly, we observe that strongly distributive elements are not well behaved even in a finite lattice. Indeed, consider Figures 6 and 7 . Observe that in Figure 6 both $s_{1}$ and $s_{2}$ are strongly distributive. But $(t \wedge a \wedge b) \vee\left(t \wedge\left(s_{1} \wedge s_{2}\right)\right)$

$$
<\left[(t \wedge a) \vee\left(t \wedge\left(s_{1} \vee s_{2}\right)\right)\right] \wedge\left[(t \wedge b) \vee\left(t \wedge\left(s_{1} \vee s_{2}\right)\right)\right] \text {, }
$$




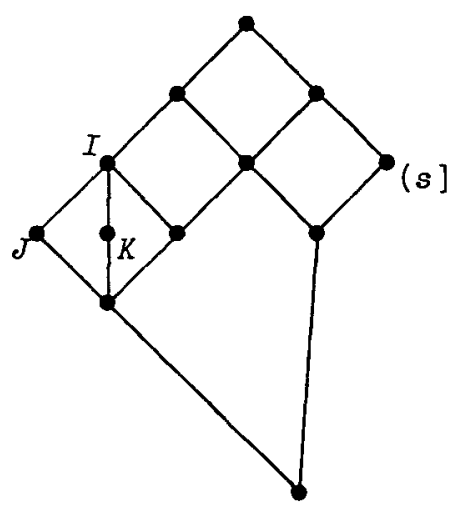

FIGURE 5

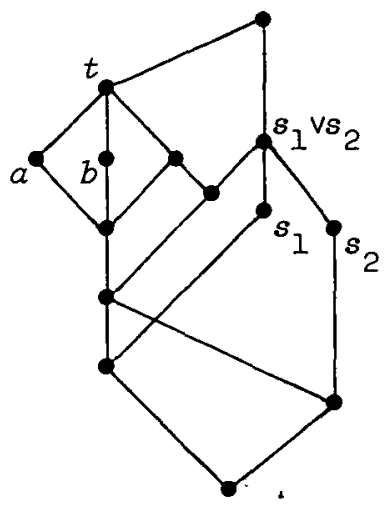

FIGURE 6 .

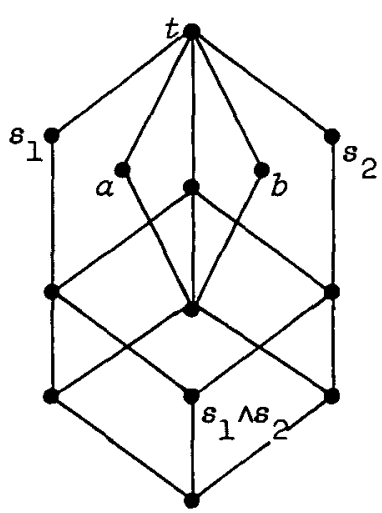

FIGURE 7 
which shows that $\boldsymbol{s}_{1} \vee \boldsymbol{s}_{2}$ is not strongly distributive. In Figure 7 , both $s_{2}$ and $s_{2}$ are strongly distributive. However,

$(t \wedge a \wedge b) \vee\left(t \wedge s_{1} \wedge s_{2}\right)$

$$
<\left[(t \wedge a) \vee\left(t \wedge s_{1} \wedge s_{2}\right)\right] \wedge\left[(t \wedge b) \vee\left(t \wedge s_{1} \wedge s_{2}\right)\right],
$$

showing that $s_{1} \wedge s_{2}$ is not strongly distributive.

\section{References}

[1] Kirby A. Baker, "Congruence-distributive polynomial reducts of lattices", Algebra Universalis 9 (1979), 142-145.

[2] Garrett Birkhoff, Lattice theory, 3rd edition (American Mathematical Society Colloquium Publications, 25. American Mathematical Society, Providence, Rhode Island, 1967).

[3] William H. Cornish, "3-permutability and quasicommutative BCKalgebras", Math. Japon. 25 (1980), 477-496.

[4] William H. Cornish, "A ternary variety generated by lattices", Comment. Math. Univ. Carolinae 22 (1981), 773-784.

[5] W.H. Cornish and R.C. Hickman, "Weakly distributive semilattices", Acta Math. Acad. Sci. Hungar. 32 (1978), 5-16.

[6] E. Fried and E.T. Schmidt, "Standard sublattices", AZgebra universalis 5 (1975), 203-211.

[7] George Grätzer, General Lattice theory (Pure and Applied Mathematics, 75. Academic Press [Harcourt Brace Jovanovich], New York, San Francisco, 1978).

[8] G. Grätzer and E.T. Schmidt, "Standard ideals in lattices", Acta Math. Acad. Sci. Hungar. 12 (1961), 17-86.

[9] Otomar Hájek, "Direct decompositions of lattices, I", Czechoslovak Math. J. 7 (82) (1957), 1-15.

[10] Robert Hickman, "Join algebras", Commm. Alg. 8(1980), 1653-1685.

[11] M.F. Janowitz, "A characterisation of standard ideals", Acta Math. Acad. Sci. Hungar. 16 (1965), 289-301. 
[12] Милан Колибиар [Mi lan Kolibiar], "Тернарная операция в структурах" [A ternary operation in lattices], Czechoslovak Math. J. 6 (1956), 318-329.

[13] Abu Saleh Abdun Noor, "Isotopes of nearlattices" (PhD thesis, Flinders University of South Australia, Bedford Park, 1980). See also: Abstract, Bull. Austral. Math. Soc. 22 (1980), $472-474$.

School of Mathematical Sciences,

Flinders University,

Bedford Park,

South Australia 5042,

Australia;

Department of Mathematics,

University of Rajshahi,

Rajshahi,

Bangladesh. 